\title{
Eine eem- und frühweichselzeitliche Abfolge im Tagebau Schöningen, Landkreis Helmstedt
}

\author{
Brigitte Urban, Harald Elsner, Adam Hölzer, \\ Dietrich Mania \& Barbara Albrecht *)
}

Geological section, Eemian, Early Weichselian, peat, travertine, silt, pollen diagrams, botanical macro remains, molluscs, paleoclimate

Kurzfassung: Im ostniedersächsischen Braunkohlengebiet bei Helmstedt waren von 1985 - 1987 im Tagebau Schöningen mächtige eeminter glaziale Bildungen aufgeschlossen. Die mit teils autochthonen, teils allochthonen Kalk-SchluffTorf-Abfolgen gefüllte, mehrere hundert Meter lange Rinne stellt das ehemalige Bett eines Baches dar, der unweit der heutigen „Mißaue" floß.

In dem Lockertravertin waren Holzreste, Früchte, Samen, Molluskenschalen und Moospolster reichlich vertreten. Die pollenanalytischen Untersuchungsergebnisse des Travertins und hangender Löß-Humuszonen spiegeln die für den nordwestdeutschen Raum bekannten Grundzüge der eem-und den Beginn der frühweichselzeitlichen Vegetationsentwicklung wider.

[An Eemian and Early Weichselian sequence of the Open Mine "Schöningen" near Helmstedt]

Abstract: In the Eastern Lower Saxony lignite area close to Helmstedt, huge deposits of Eemian age have been exposed during 1985 - 1987. A channel of several hundred meters of extend, filled with autochtonous and partly allochtonous carbonate-silt-peat sequences, might reflect the early riverbed of a creek, running only a few meters beside the nowaday brook "Mißaue".

*) Anschriften der Autoren: Prof. Dr. BrigitTe UrbanKƯTTEL und BARBARA AlBRECHT, Fachhochschule Nordostniedersachsen, Herbert-Meyer-Straße 7, 3113 Suderburg.

Dipl.-Geol. Harald ElSNER, Institut für Geologie und Paläontologie der Universität Hannover, Callinstraße 30 , 3000 Hannover 1.

Dr. ADAm HÖlzer, Staatliches Museum für Naturkunde Karlsruhe, Postfach 3949, 7500 Karlsruhe.

Dr. habil. D. MANIA, Landesmuseum für Vorgeschichte, Richard-Wagner-Straße 9/10, $4020 \mathrm{Halle} /$ Saale.
The loose kind of travertine sediments were rich in woodremains, fruits, seeds, shells of molluscs and mosses. The pollenanalytic results reflect the characteristic features of the Eemian and the beginning of the Early Weichselian vegetation development.

\section{Einleitung}

Im zentralen Teil des subherzynen Beckens sind in Ost-Niedersachsen (Abb. 1) tertiäre Lockersedimente weit verbreitet. Sie sind an Flankenbereiche der Sattelstrukturen aus Zechsteinsalzen gebunden, deren längste der $80 \mathrm{~km}$ lange Helmstedt-Staßfurter Sattel ist. In seinen Muldenbereichen sind mächtige eozäne Braunkohlenflöze erhalten, die heute von der Braunschweigische Kohlen-Bergwerke AG (BKB) abgebaut werden. Die im Hangenden der Tertiär-Schichten weitflächig auftretenden, verschiedenartigen, teilweise lückenlosen Quartär-Abfolgen (Abb. 2) sind

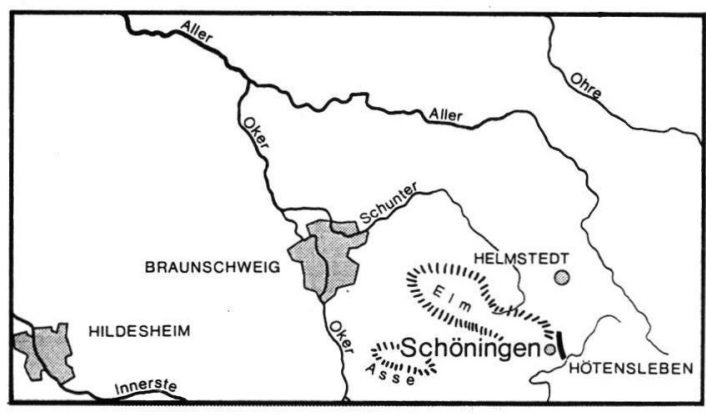

Abb. 1: Lage des Tagebaus „Schöningen” im nördlichen Harzvorland, östlich der Stadt Schöningen. 
seit 1983 Untersuchungsgegenstand des Projekts "Archäologische Schwerpunktuntersuchungen im Helmstedter Braunkohlerevier" (ASHB), das vom Institut für Denkmalpflege (Hannover) zusammen mit der Fachhochschule Nordostniedersachsen (Suderburg) durchgeführt wird (THIEME, MAIER \& URBAN 1987). Seit 1986 wurden in Zusammenarbeit mit dem Institut für Geologie und Paläontologie der Universität Hannover die Quartär-Deckschichten intensiver untersucht (ELSNER 1987; HARTMANN 1988; LENHARD 1989), deren inzwischen überregionale Bedeutung nicht zuletzt in einer durch die Deutsche Forschungsgemeinschaft seit 1988 großzügig gewährten Projektunterstützung zum Ausdruck kommt.
Inhalt dieses Beitrages ist die über $5 \mathrm{~m}$ mächtige, eem- und frühweichselzeitliche Abfolge im nördlichen Baufeld Esbeck des Tagebaues Schöningen (Abb. 1 und 2), die zwischen den Jahren 1985 und 1987 gut aufgeschlossen war.

\section{Ausbildung und Genese eemzeitlicher Sedimente im Tagebau Schöningen der BKB AG, Helmstedt}

Während im Südteil des Tagebaues Schöningen (Baufeld Schöningen) limnisch-telmatische Ablagerungen des Eem nur aus Bohrungen bekannt sind, waren

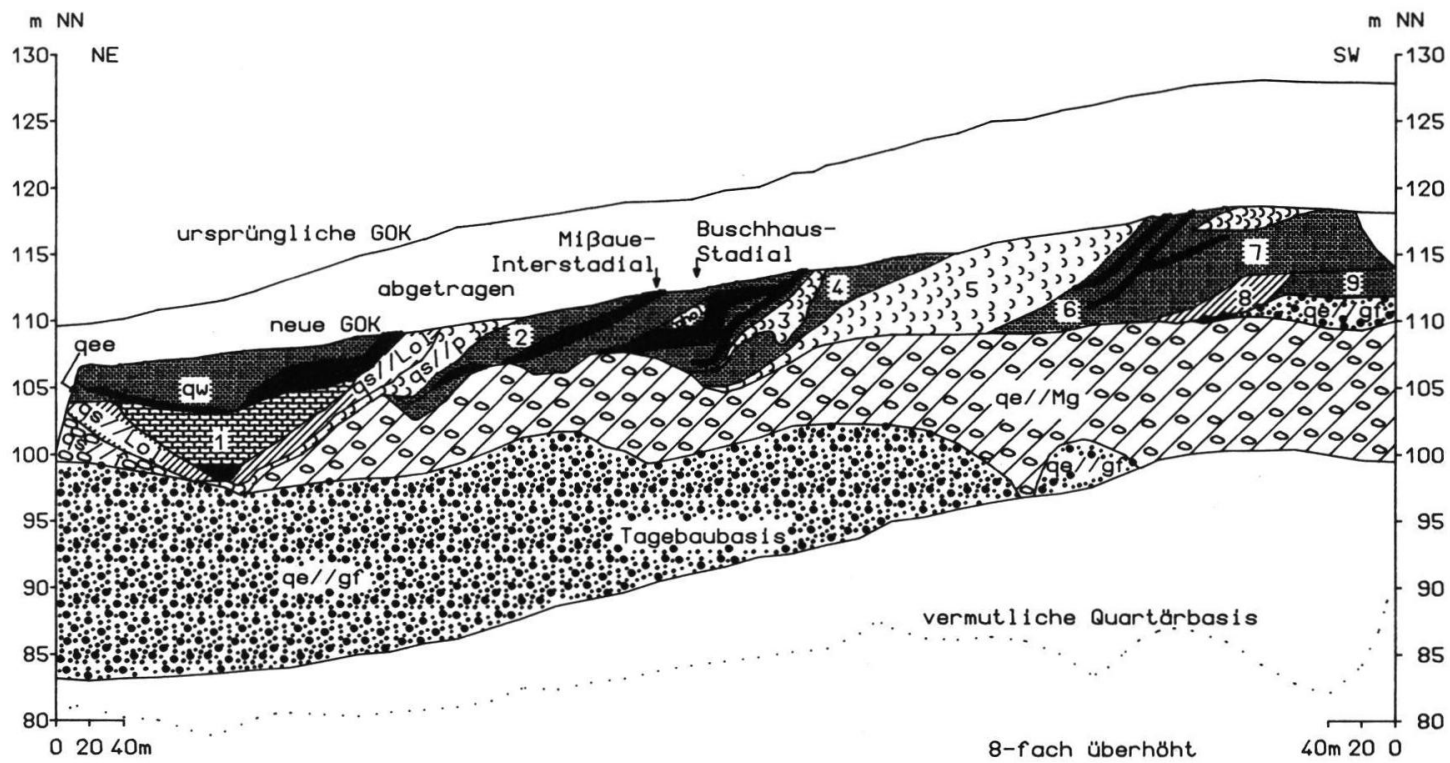

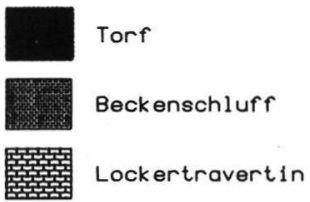

1 Eem-Travertin

2 Fuhne B

3 Fließerde

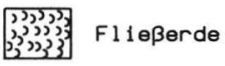

응요 Bach-Sedimente

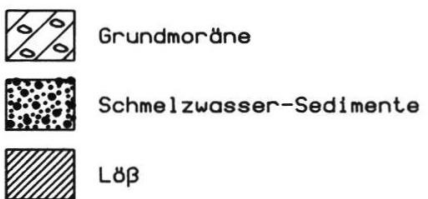

7 Offleben I-Interstadial

8 Elster-Schwemml $6 \beta$

9 Elster-Beckenschluff/Warwite

Abkürzungen :

$$
\begin{aligned}
& \text { qe }=\text { Quartär Elster - Kaltzeit } \\
& \text { qhol }=\text { Quartär Holstein - Warmzeit } \\
& \text { qs }=\text { Quartär Saale }- \text { Kaltzeit } \\
& \text { qee }=\text { Quartär Eem - Warmzeit } \\
& \text { qw }=\text { Quartär Weichsel }- \text { Kaltzeit }
\end{aligned}
$$

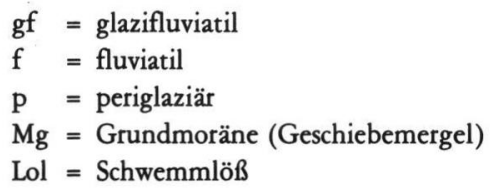

Abb. 2: Profilschnitt durch die quartären Schichtfolgen im Tagebau „Schöningen”, Landkreis Helmstedt (Stand Ende 1987), Aufschlußlänge ca. 1 km; TK 25: Nr. 3831, 3832. (Aus: UrbAN et al. 1988). 
mächtige Sedimente dieses Interglazials im Nordteil des Tagebaus (Baufeld Esbeck) über längere Zeit hinweg aufgeschlossen (Abb. 2). Es handelte sich dabei um humusreiche Ablagerungen, die eine ca. $200 \mathrm{~m}$ breite, bereits saalezeitlich angelegte Rinne, ausfüllten. Diese Rinne wurde vermutlich durch Schmelzwässer des stagnierenden Drenthe-I-Inlandeises geformt. Im Untersuchungsjahr 1987 waren Reste einer Drenthe-Grundmoräne nicht nachweisbar. Seit 1988 sind allerdings aufgrund neuer Aufschlußbedingungen (HARTMANN 1988; LENHARD 1989) wiederholt drenthezeitliche Moränenreste aufgefunden und näher untersucht worden. Saalezeitliche solifluidale Ablagerungen, deren Ursprungsgebiet in den $\mathrm{Mu}$ schelkalkhängen des Elms zu suchen sind, sind weit verbreitet (Abb. 2). Teilweise sind diese saalezeitlichen Fließerden erneut umgelagert worden. Im Hangenden findet sich ein maximal $3 \mathrm{~m}$ mächtiger, gelbbrauner, stark kryoturbat überprägter Schwemmlöß, mit (ELSNER 1987) grünlicher, auf Reduktion hindeutender Farbgebung und starker Durchwurzelung seines Dachbereiches. Mit diesem wahrscheinlich warthezeitlichen Schwemmlöß findet die saalezeitliche Abfolge ihren Abschluß. Im Hangenden dieser SaaleSerie trat 1987 ein ca. $50 \mathrm{~cm}$ starker „Basistorf” auf, der zum Zeitpunkt der Erstuntersuchungen von MERKT (1986) noch nicht aufgeschlossen war. Mit scharfer Untergrenze lag der Torf dem grünlichen Schluff auf. Auffällig waren bis zu $1 \mathrm{~m}$ lange, regellos verteilte Baumstämme. Vereinzelt waren große zertrümmerte Gastropodenschalen sichtbar. Lateral zu den Rinnenrändern verzahnte sich der beschriebene Basistorf mit schwarzem, stark humosem Beckenschluff. Im Hangenden folgte ein bis $4 \mathrm{~m}$ mächtiger, mürber, zum Teil gastropodenreicher, weißgrauer Süßwasserkalk, wie er auch aus dem nahegelegenen Elmgebiet mit ebenfalls eemzeitlichem Alter erstmals von HUCKRIEDE (1967) beschrieben wurde. Beim Ausschlämmen dieses Lockertravertins blieben Holzrestchen, Kalkschluff, Travertinbröckchen, Quarzgrobsandkörner, Früchte, Gastropodenschalen und wenige Bivalvenschalen übrig.

Stellenweise wurden in der aufgeschlossenen Travertinbank linsenförmig angereicherter Feinsand und Schluff zur Hauptkomponente, an anderer Stelle waren Torffetzen, -linsen und -flasern in Lagen zusammengeschwemmt. Erwähnenswert sind weiterhin noch chlorophyllhaltige Quellmoosreste sowie vollständig erhaltene große Gastropodenschalen.

Einzelne Travertinbänke sind verschiedenen Befunden nach autochthon entstanden, während andere Sedimentpakete partiell als resedimentiertes Umlagerungsprodukt der näheren Umgebung zu deuten sind. Der Kalkgehalt stammt aus Karbonaten des Elms. An Quellen und Grundwasseraustritten inner- halb der eemzeitlichen Rinne ist er zum Teil biogen wieder ausgefällt worden (vgl. KOERT \& DIENEMANN 1927: 49). Die Rinne selber konnte anhand ihrer charakteristischen Füllung durch Auswertung zahlreicher Bohrprotokolle und durch Beobachtungen von LUUTGE (1984) über eine Länge von mehreren $\mathrm{km}$ rekonstruiert werden. Sie scheint das Bett eines eemzeitlichen Baches darzustellen, der zumindest im Baufeld Esbeck, weniger als $100 \mathrm{~m}$ von der heutigen Mißaue entfernt, floß

Überlagert wird der Lockertravertin dieser „Ur-Mißaue" von einem relativ dünnen Torfbändchen, das im Gegensatz zu den Lockertravertinschichten im Liegenden bis an die Rinnentänder zu verfolgen war. Es war stark zersetzt und führte vereinzelt Lagen aus Kalkschluff. Im Hangenden dieses Torfes folgten mehrere Meter humoser Kalkschluff. Wurzelröhren und lagige, orangebraune Marmorierung deuten auf Bodenbildungsprozesse hin, die in den Grenzbereich Eem-Weichsel einzuordnen sind.

In den mächtigen weichselzeitlichen Löß waren an einer Stelle im Hangenden des Eem-Travertins $\mathrm{Hu}$ musschichten eingeschaltet, die, den pollenanalytischen Untersuchungen zufolge, in die frühe WeichselKaltzeit zu stellen sind.

\section{Vegetations- und Sedimententwicklung während der Lockertravertinbildung und des Früh-Weichsels}

\subsection{Profilaufbau}

Das Interglazialprofil (Abb. 2) wurde über seine Gesamtmächtigkeit von $270 \mathrm{~cm}$ sedimentologisch und pollenanalytisch untersucht, das hangend aufgeschlossene Früh-Weichsel über $250 \mathrm{~cm}$ Mächtigkeit.

\section{Ablagerungen des Eem:}

Zwischen $0 \mathrm{~cm}$ und $170 \mathrm{~cm}$ beträgt der Kalkanteil des Lockertravertins zwischen $29 \%$ und $89 \%$. Dieser lockere Mergel ist teilweise von humosen Einschaltungen unterbrochen, auch das Liegende ist teilweise humos bei sehr schlechter Pollenerhaltung.

Bei $180 \mathrm{~cm}$ endet die Karbonatführung und darüber folgt ein toniger Schluff. Dieser ist stellenweise humos, sein Einsetzen markiert jedoch bereits das Ende des Interglazials.

\section{Ablagerungen des Früh-Weichsels:}

Der basale Profilbereich $(250 \mathrm{~cm}$ bis $180 \mathrm{~cm})$ enthält zwischen 2,3\% und 0,6\% organischen Kohlenstoff, 


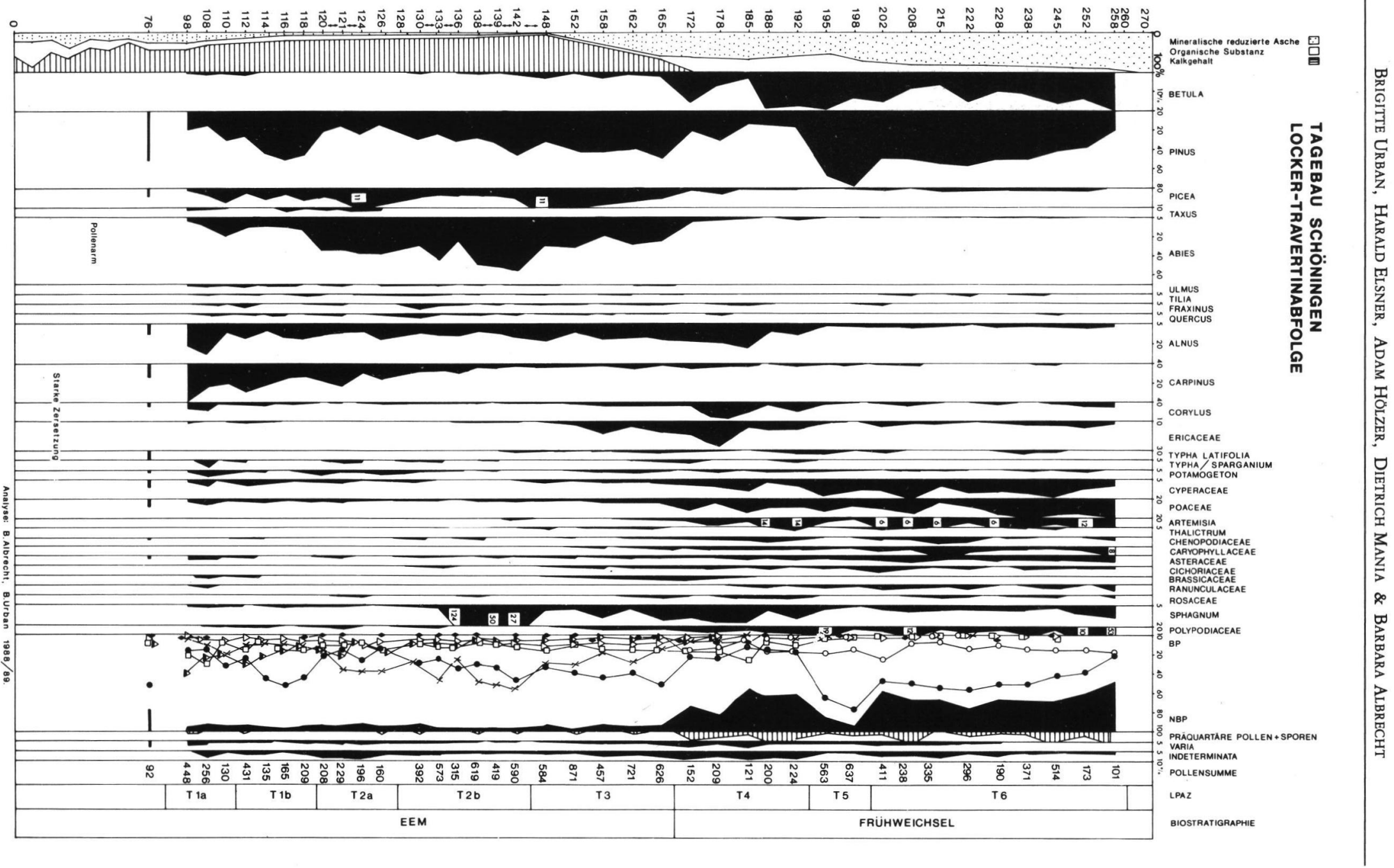


ist entkalkt und weist niedrige $\mathrm{pH}$-Werte auf. Die nachfolgenden, jüngeren Profilabschnitte sind partiell schwach humos und schwach kalkhaltig. Tabelle 1 zeigt die Korngrößenverteilung.

Tab. 1: Korngrößenzusammensetzung im Profil SFW (Früh-Weichsel)

$$
\begin{aligned}
& \text { 190-180 cm: } 93 \% \text { Schluff, } 7 \% \text { Sand = Schluff } \\
& 150-140 \mathrm{~cm}: 19 \% \text { Ton, } 78 \% \text { Schluff, } \\
& 3 \% \text { Sand } \\
& =\text { toniger Schluff } \\
& \text { 110-100 cm: } 35 \% \text { Ton, } 65 \% \text { Schluff = stark schluffiger } \\
& 70-60 \mathrm{~cm}: 52 \% \text { Ton, } 46 \% \text { Schluff, } \\
& 2 \% \text { Sand } \\
& \text { Ton } \\
& =\text { schluffiger } \text { Ton } \\
& 30-20 \mathrm{~cm}: 23 \% \text { Ton, } 72 \% \text { Schluff, } \\
& 5 \% \text { Sand } \\
& =\text { toniger Schluff }
\end{aligned}
$$

\subsection{Paläoökologische Verhältnisse}

\subsubsection{Methodisches Vorgehen}

Die Pollenaufbereitung erfolgte in einer neuerdings abgewandelten Form ohne Schweretrennung (URBAN 1978) in folgender Weise:

1. Kalkzerstörung

2. $\mathrm{KOH}-$ Behandlung $(10 \% \mathrm{KOH}, 2-10$ Minuten)

3. HF-Behandlung (HF konz., kalt über Nacht)

4. Siebung mit Ultraschallbad (10 $\mu \mathrm{m}$ Sieb) Fa. Branson

5. Acetolyse (ERDTMANN 1960).

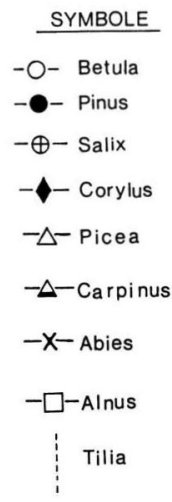

Abb. 3: Pollendiagramm der Lockertravertinabfolge.
Zur Berechnung des Gesamtpollendiagrammes und jedes einzelnen Taxons wurde die Basissumme aus den Baumpollen, den terrestrischen Nichtbaumpollen inklusive Gräserpollen, jedoch ohne Sauergräserpollen (Cyperaceae) gebildet.

Die Großrestwerte sind auf eine Basissumme aller Großreste $=100 \%$ bezogen .

\subsubsection{Pollenanalytische Gliederung der eemzeitlichen Abfolge}

Das gesamte Pollendiagramm (Abb. 3) ist in sechs fortlaufende Pollenzonen (Local pollen assemblage zones, LPAZ) und teilweise weiter in Subzonen gegliedert worden. Der basale Bereich des Profils $(0-76 \mathrm{~cm})$ ist pollenarm und daher statistisch nicht auswertbar. Dennoch lassen sich Rückschlüsse auf starke Pollenzersetzung und zum Teil turbulente Ablagerungsbedingungen ziehen. Eine Einzelprobe bei $76 \mathrm{~cm}$ Tiefe ist aufgrund der geringen Pollensumme nur begrenzt auswertbar. Die Kiefer herrscht bei gleichzeitigem Vorkommen von Hainbuche, Hasel und Erle vor. Die eigentliche Travertinbildung und damit die Sedimentation der interglazialen Ablagerungen, setzt demnach erst relativ spät im Interglazial ein.

Zone T1: Carpinus-Pinus-Zone, gegliedert in zwei Subzonen

Subzone T1a $(98-111 \mathrm{~cm})$ :

Carpinus-Pinus-Alnus-Subzone

In dieser Zone erreicht die Hainbuche ihre höchsten Werte (bei $40 \%$ ). Erle und Kiefer folgen mit Werten um $20 \%$. Auch die Hasel weist noch Anteile um 5\% auf. Der Eichenmischwald hat kaum Bedeutung innerhalb des Waldbildes. Vereinzelt treten in diesem und in dem nächstjüngeren Unterabschnitt Pollen von Taxus und Buxus auf. Pollen krautiger Pflanzen sind nur gering vertreten. Pollen aquatischer Pflanzen wie Typha/Sparganium und Potamogeton kommen in diesem und den nächstjüngeren Abschnitten häufig vor. Die Grenze zur Subzone T1b wurde an dem Überschneidungsbereich der Kiefern- und Hainbuchenkurve gezogen.

\section{Subzone T1b $(111-118 \mathrm{~cm})$ : Pinus-Carpinus-Picea-Alnus-Subzone}

Die Kiefer hat deutlich zugenommen, die Hainbuchenkurve ist dagegen etwas rückläufig. Weiterhin sind Fichte und Erle gut repräsentiert. Die Eichenmischwaldelemente haben ebenso wie die Hasel eine 
sehr geringe Beteiligung. Die Grenze zur nächstjüngeren Pollenzone wurde in den Bereich der stark abfallenden Kiefern- und allmählich ansteigenden Tannenkurve gelegt.

Zone T2: Abies-Pinus-Zone, ebenfalls in zwei Subzonen untergliedert

Subzone T 2a $(118-127 \mathrm{~cm})$ : Abies-Pinus-Carpinus-Picea-Subzone

Die Kiefernwerte sind abgesunken, die Tanne erreicht vorübergehend Werte um $40 \%$ und dominiert auch über die Hainbuche und die Fichte. Pollen aquatischer Pflanzen treten sporadisch auf. Kräuterpollen terrestrischer Pflanzen fehlen weitgehend.

Die Grenze zum Abschnitt T 2b wurde in den Bereich der sich überschneidenden Tannen- und Kiefernkurve gelegt.

Subzone T 2b $(127-145 \mathrm{~cm})$ : Abies-Pinus-Subzone

Bei deutlich abgesunkenen Fichtenwerten erreicht die Tanne ihre Maximalausbreitung mit einem Gipfel um $60 \%$. Auch die Hainbuche ist auf Werte deutlich unter $10 \%$ gefallen. Diese nehmen während des weiteren Interglazialverlaufes auch nicht wieder erheblich zu. Gegenüber einem mengenmäßig immer noch unbedeutenden Anteil terrestrischer Kräuterpollen sind in diesem Abschnitt inbesondere die SphagnumWerte zu erwähnen. In diesem Horizont konnten auch Reste von Moosrasen ausgemacht werden. Die Grenze zum nächstjüngeren Abschnitt wurde im Bereich deutlich fallender Tannenwerte und zunehmender Fichtenanteile gelegt.

\section{Zone T $3(145-168 \mathrm{~cm})$ : Pinus-Picea-Abies-Zone}

Die Kiefer dominiert in dieser Zone mit Werten um $40-50 \%$ über die Tanne, deren Anteile um $30 \%$ herum liegen, und die Fichte. Letztere hat zu Beginn dieser Zone ein zweites Maximum, das bei $11 \%$ liegt. Ein erster deutlicher Anstieg der Ericaceae-, der Poaceae- und der Cyperaceae-Kurven läßt sich in der zweiten Hälfte des Abschnittes feststellen; auch das Spektrum der terrestrischen Kräuter wird zunehmend typenreicher und nimmt auch quantitativ zu.

Die Grenze zum sich anschließenden Abschnitt T4 wurde in den Bereich der zunehmenden Nichtbaumpollenanteile und der ansteigenden Birkenkurve gelegt.
Zone T4 $(168-190 \mathrm{~cm})$ :

\section{Betula-Pinus-Nichtbaumpollen-Zone}

Mit dieser Pollenzone beginnt die Sedimentation eines kalkfreien, an der Basis noch humosen tonigen Schluffes. Das Pollenspektrum zeigt das Ende des Interglazials an. Die Birkenkurve ist stark angestiegen, wird jedoch überwiegend von der der Kiefer überlagert. Die Ericaceae erreichen Werte um $28 \%$, Poaceae und Cyperaceae zeigen erhöhte Werte. Wiederum sind die Sphagnen stark vertreten. Innerhalb der terrestrischen Kräuter fallen vor allem die beiden Artemisia-Gipfel mit $14 \%$ am Ende dieser Pollenzone auf. In diesem Abschnitt gibt es aufgrund der geänderten Sedimentationsart und -bedingungen eine Reihe von Störungen, die sich in einem unruhigen Diagrammverlauf widerspiegeln.

Auf partielle Umlagerung von Pollen und Sporen deutet auch die Kurve der präquartären Sporomorphen hin. Die auch in Zone T4 durchgeführten Betula-Pollengrößenmessungen weisen einen erheblichen Anteil von Betula-Körnern mit einem Durchmesser von $22 \mu \mathrm{m}$ auf (von Wand zu gegenüberliegender Pore gemessen). Demnach dürften noch überwiegend baumförmige Birken existiert haben.

Die Grenze zum folgenden Abschnitt Ts wurde in den Bereich des Steilanstieges der Kiefernkurve gelegt.

\section{Zone Ts $(190-200 \mathrm{~cm})$ : Pinus-Betula-Zone}

Diese Pollenzone ist mit nur zwei Proben erfaßt worden. Es finden sich kaum noch Pollen thermophiler Gehölze, auch der Anteil an umgelagerten präquartären Sporomorphen ist abgesunken. Demgegenüber haben die Kiefern- und Birkenanteile deutlich zugenommen. Die Nichtbaumpollenanteile fallen vorübergehend auf Werte um $10 \%$ von vorher $40-$ $50 \%$ im Abschnitt T4, ab. Die Betula-Größenmessungen zeigen bei $20 \mu \mathrm{m}, 22 \mu \mathrm{m}$ und bei $24 \mu \mathrm{m}$ Verteilungsschwerpunkte, die kleinen Formen sind nur mit geringen Prozentanteilen vertreten (Abb. 4). Es dominieren die baumförmigen Birken (Abb. 5). Wieder zunehmende Kräuteranteile markieren die Grenze zum Abschnitt T 6.

\section{Zone T 6 (200-258 m): \\ Pinus-Betula-Nichtbaumpollen-Zone}

Erneut ist eine starke Zunahme der Poaceae, der Cyperaceae und der lichtliebenden krautigen Pflanzen festzustellen. Innerhalb der Baumpollen herrscht die Kiefer mit Werten um $60 \%$ vor. Die Birkenanteile liegen bei $15 \%$. Jetzt treten auch innerhalb 


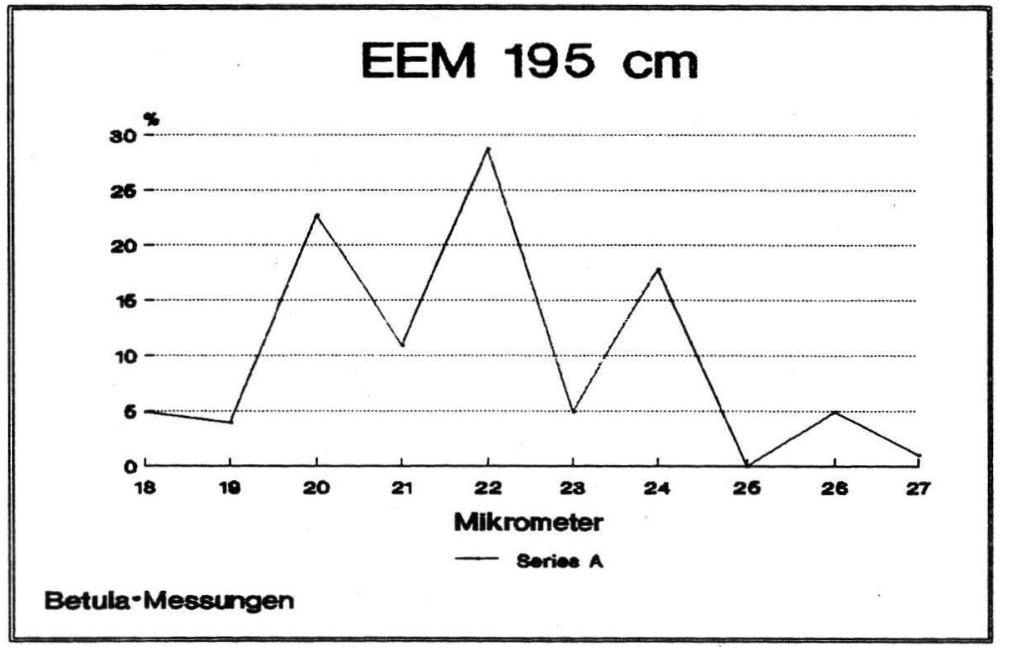

Abb. 4: Betula-

Pollengrößenmessungen.

der vermessenen Betula-Pollenkörner vermehrt die kleinen Formen auf. In dieser Zone wurde Betula nana auch durch Makroreste nachgewiesen (Abb. 5). Artemisia erreicht Höchstwerte von $12 \%$, daneben haben Caryophyllaceae, Chenopodiaceae und Asteraceae partiell deutlich erhöhte Werte.

\subsubsection{Gliederung des Großrestdiagramms}

Im unteren Teil des Profils (Abb. 5: Abschnitt 1) sind nur wenige Pflanzenreste bestimmbar. Es handelt sich um Radizellentorfe. Erst ab der Probe $85 \mathrm{~cm}$ (Abschnitt 2) tritt dann plötzlich Tomenthypnum nitens als dominierender Bestandteil auf. Die folgenden Abschnitte 3 und 4 umfassen nur wenige Zentimeter und enden im Sphagnum-Torf. Der sich anschließende Abschnitt 5 ist wieder durch Radizellentorfe charakterisiert. Es nehmen aber auch die Holzreste und Samen zu. Ebenso finden sich Potamogeton-Steinkerne. Eine sichere Bestimmung war aber nicht möglich, da der Erhaltungszustand sehr schlecht war. In Abschnitt 6 setzen wieder wenige Moose ein.

\subsubsection{Interpretation der Großreste}

Großrestanalysen geben einen Überblick über die Vegetation direkt an der Profilstelle oder in der nächsten Umgebung. Außer in Fließgewässern werden sie meist nur über eine geringe Entfernung transportiert. Dies gilt besonders für die Moose. Bei ihnen läßt sich im Gegensatz zu Samen und Früchten oft ausschließen, daß sie über eine weitere Entfernung transportiert wurden. Damit ermöglichen sie eine Rekonstruktion der lokalen Verhältnisse. Oft erlauben die Großreste auch eine weitergehende Artbestimmung als die Pollen.
Der unterste Abschnitt (1) zeigt die Reste eines typischen Niedermoores mit Cyperaceenresten, vor allem Radizellen. Danach kommt es zu einer Auflichtung der Vegetation, wobei Tomenthypnum nitens zur dominierenden Art wird. Es ist charakteristisch für mehr oder weniger kalkreiche, nasse bis feuchte Niedermoore, aber auch für die Dryas-Heide (Dickson 1973). Die Art kann auch mit Torfmoosen vergesellschaftet sein, so zum Beispiel mit Sphagnum warnstorfii, Sphagnum palustre oder Sphagnum subsecundum.

Im folgenden Abschnitt (2) treten Torfmoose und Paludella squarrosa hinzu. Paludella squarrosa ist leicht zu erkennen an ihren squarrösen, spitzen Blättern. Nach GEISSLER \& ZOLLER (1978) gehört die Art mit Sphagnum warnstorfii, Sphagnum teres und Drepanocladus revolvens zu den weniger anspruchsvollen Niedermoorarten. Es kann sogar mit ombrotraphenten Arten wie Andromeda polifolia, Vaccinium oxycoccus oder Drosera rotundifolia vergesellschaftet sein. GEISSLER \& ZOLLER (1978) geben einen Überblick über das heutige Vorkommen der Art im westlichen Alpenraum und ihre Vergesellschaftung.

Die umfangreichste Darstellung der Standortansprüche und Vergesellschaftung von Paludella squarrosa findet sich bei RYBNIČEK (1966). Danach hat die Art ein Optimum auf Torf bei einem Grundwasserstand zwischen -8 und $-11 \mathrm{~cm}$ und erträgt keine Überflutung. In seinem Untersuchungsgebiet liegen die $\mathrm{pH}$-Werte zwischen 4,7 und 6,7. Die Calcium-Gehalte bewegen sich zwischen 5,6 und $21,5 \mathrm{mg} / \mathrm{l}$ und der Magnesiumgehalt zwischen 0,9 und $5,8 \mathrm{mg} / \mathrm{l}$. Die Schwankungsbreite der Werte ist sehr weit. RYBNIČEK gibt Werte von $0,8-120 \mathrm{mg}$ Calcium pro Liter und $\mathrm{pH}$-Werte zwischen 6 und 8 an. Diese große Schwankungsbreite ist in dem relativ großen Abstand 


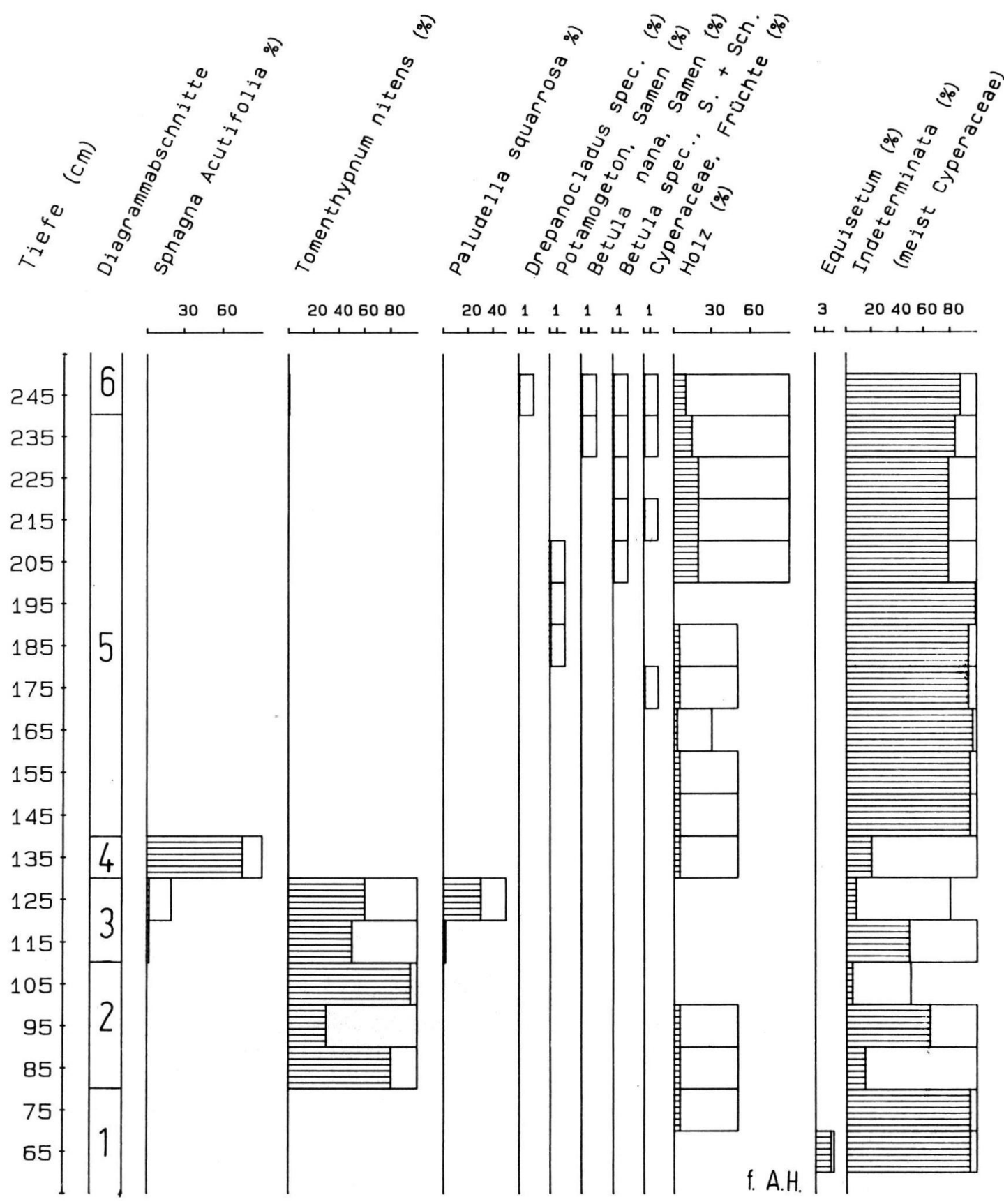

Abb. 5: Großrestdiagramm. 
zwischen dem lebenden Moos und dem Grundwasser begründet.

Interessanterweise konnten auch im Übergangsbereich keine Blätter von Spagnum warnstorfii oder anderen mehr minerotraphenten Torfmoosen gefunden werden.

Im Bereich des Maximums von Paludella befindet sich bereits der Übergang zum Hochmoor, das hier mit der Probe $135 \mathrm{~cm}$ erfaßt wird (Abb. 5). Im Pollendiagramm ist dieser Abschnitt durch erstmals stark ansteigende Sphagnumsporenwerte (LPAZ T 3, Abb. 3) charakterisiert. Eine Entscheidung, ob es sich um Sphagnum rubellum oder S. nemoreum handelt, war nicht möglich, da keine Stammblätter erhalten waren. $\mathrm{Da}$ Sphagnum nemoreum normalerweise an trockneren Standorten steht als S. rubellum, hätte man etwas mehr über die lokalen Feuchtigkeitsverhältnisse aussagen können. Man kann aber aus der Entwicklung ombrotropher Verhältnisse ablesen, daß ein gewisser Überschuß an Niederschlägen vorhanden gewesen sein mußte, um überhaupt eine Hochmoorbildung zu ermöglichen.

Die Sphagnumtorfe reißen plötzlich ab. Die Fläche wird wohl überschwemmt. Es finden sich Steinkerne von Potamogeton, aber auch Birkensamen, die aber sehr schlecht erhalten sind. Einige können aber sicher Betula nana zugeordnet werden. Diese deuten das Ende der Warmphase an. Erst am oberen Ende des Profils finden sich wieder Moose als Zeiger für niedrigen Wasserstand wenigstens der Umgebung.

BEHRE \& LADE (1986) sowie BEHRE (1989) zeigen ähnliche Großrestabfolgen für das Eem, das Odderadeund das Oerel-Interstadial, die auch bis in den Sphagnumtorf und damit wohl das Hochmoor führen.

\subsubsection{Molluskenvergesellschaftung des Eem-Profils}

Aus der eeminterglazialen Serie wurden zwei Proben hinsichtlich ihrer Molluskenführung entnommen und ausgeschlämmt. Die eine aus dem mergeligen Locker- travertin, die andere aus dem Torflager, das die Serie nach oben abschließt.

Dem relativ geringen Probenumfang entspricht der Fossilinhalt: aus dem Lockertravertin liegen etwa 200 bestimmbare Exemplare vor, aus dem Torf sind es etwa 20. Die erstgenannte Probe läßt sich noch statistisch auswerten, obwohl über die bestimmbaren Exemplare hinaus zahlreiche Schalenbruchstücke vorkommen.

\subsubsection{Die Molluskenfauna aus dem Lockertravertin}

Insgesamt wurden in der Thanatozönose 20 Arten nachgewiesen (Tab. 2). Die Pisidien wurden nicht näher bestimmt. Es handelt sich um Arten, die auch heute in Mitteleuropa vorkommen. Sie wurden nach der Methode von LoŽEK (1964) und MANIA (1973) ökologischen Gruppen zugewiesen und hinsichtlich ihrer Ansprüche paläoökologisch ausgewertet.

Neben 16 terrestrischen Arten (Gruppe 1 bis 9) kommen, einschließlich der Pisidien, mindestens 5 aquatische Arten vor (Gruppe 10). Nach ihrer individuellen Häufigkeit umfassen letztgenannte $21,1 \%$. Sie sind mehr oder weniger als autochthone Komponente der Assoziation aufzufassen. Dies entspricht auch dem Sediment aus dem sie stammen. Jedoch ist zu vermuten, daß ebenso ein großer Teil der Gruppe 2 (47,6\%) sowie die einzige Art der Gruppe 3, die immerhin $8,8 \%$ erreicht, aufgrund ihrer großen Häufigkeit zur autochthonen, ehemals am Einbettungsort lebenden Fauna gehörten. Diese Verhältnisse verdeutlicht das Individuenspektrum (Abb. 6).

10 Arten sind an Wald gebunden. Fünf davon stellen ausgesprochene Waldarten dar (Gruppe 1), die an feuchten Standorten im thermophilen Laubmischwald leben - außer Discus ruderatus, der auch in reinen Nadelwäldern sowie heute in Mitteleuropa meist in der montanen bis subalpinen Stufe vorkommt. Die Arten der Gruppe 2 sind nicht streng an geschlossene Wälder gebunden; sie kommen auch an gedeckten, schattigen, meist mäßig feuchten

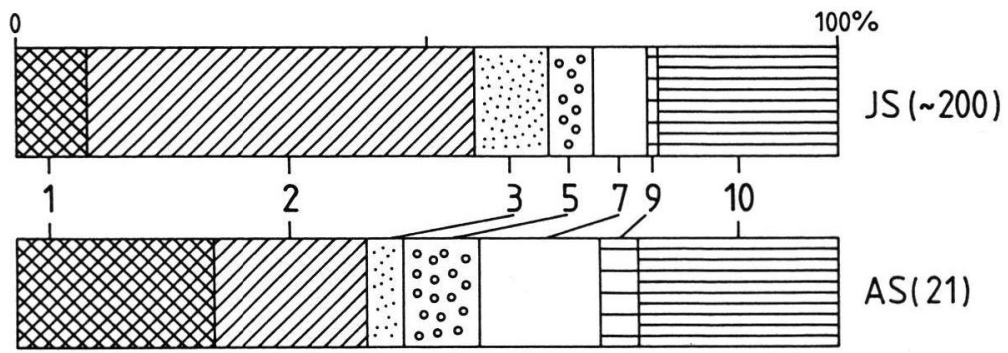

Abb. 6: Molluskenfauna der Lockertravertinabfolge (Probe 1).

JS Individuenspektrum, AS Artenspektrum, $1-10$ ökologische Gruppen nach LOŽEK 1964. 
Stellen im offenen Gelände vor, so zum Beispiel in Gebüschfluren. Clausilia pumila (Gruppe 3) lebt in feuchten bis sehr feuchten Wäldern der Auen und Tiefebenen.

Arten der offenen Landschaft sind selten. Neben Pupilla muscorum, die nur in wenigen Exemplaren nachgewiesen wurde, erscheint häufiger Vallonia costata. Aber diese kann auf mäßig feuchten Wiesen leben und dringt in die Waldlandschaften vor.

Alle anderen terrestrischen Arten sind euryök und haben mehr oder weniger hohe Feuchtigkeitsansprüche.

Unter den Wasserarten treten neben Arten der stehenden bis mäßig bewegten Gewässer einige Sumpfarten auf.

Die nachgewiesene Fauna ist nach den ökologischen Ansprüchen der einzelnen Arten relativ einheitlich. Das geht auch aus dem Artenspektrum hervor (Abb. 6). Es handelt sich um die Fauna einer kleineren Aue, die mit Wald und Gebüsch auf feuchten bis sehr feuchten oder sumpfigen Standorten dicht bestanden war. Nennenswerte offene Standorte können nicht nachgewiesen werden, höchstens in Form sumpfiger Wiesen. Die Aue wurde von einem kleineren Fließgewässer durchzogen, sonst waren Sümpfe und kleine flache, stehende Gewässer ausgebildet.

Die Assoziation zeigt durchschnittliche warmzeitliche Verhältnisse an. Sie enthält einige bezeichnende Arten der feuchtwarmen Zeitabschnitte des Interglazials. Allerdings fehlen sogenannte hochwarmzeitliche Leitarten, die im Vergleich mit der Rezentfauna des Untersuchungsgebietes exotischen Charakter haben, wie zum Beispiel Helicigona banatica. So liegt eine allgemeine mitteleuropäische Laubmischwald-Gesellschaft vor. Das zeigt auch ihre Zusammensetzung nach den Arealverhältnissen der einzelnen Arten in der Gegenwart (vgl. Tab. 2).

Die Fauna deutet auf warm-gemäßigte Klimaverhältnisse. Bezeichnende Warmzeitarten sind Orcula doliolum, Acanthinula aculeata, Aegopinella nitens, Vitrea subrimata, Ceaea hortensis, Discus rotundatus, Laciniaria biplicata. Orcula doliolum und Vitrea subrimata haben heute ihren Verbreitungsschwerpunkt außerhalb Mitteleuropas. Beide sind meridional im nördlichen Mittelmeergebiet verbreitet. Häufiger waren sie nach Aussage der holozänen Molluskensukzessionen (MANIA 1973) in den wärmeren Zeitabschnitten des Holozäns, im Atlantikum und späten Boreal. Sie weisen auch in der vorliegenden Fauna auf relativ warme Verhältnisse im Sinne von noch warm-temperiertem Klima hin.

\author{
3.2.4.2 Die Molluskenfauna \\ aus dem spätinterglazialen Torflager
}

Nur einige Arten sind in der individuenarmen Fauna nachweisbar (s. Tab. 2): Bradybaena fruticum und Arianta arbustorum (Gruppe 2) sowie etwa doppelt bis dreimal so häufig wie diese Clausilia pumila (Gruppe 3). Diese Arten sind keine Leitarten der Warmzeiten, sie kommen auch in den kühleren Übergangsabschnitten zu den Kaltzeiten vor. Sie verweisen - wie der Torf - auf sumpfige und feuchte Auen mit Baumbestand und Gebüsch, durchsetzt von offenen Standorten.

\subsubsection{Interpretation der Molluskenanalyse}

Die über die Molluskenfauna erhaltenen Ergebnisse decken sich im Wesentlichen mit denen der Palynologie. Demnach war das Klima zwar warm, jedoch herrschten keine hochwarmzeitlichen Klimaverhältnisse mehr, diese sind bereits überschritten.

Ähnliche, an heutige faunistische Verhältnisse erinnernde Gesellschaften ohne nennenswerte exotische Arten, wurden auch in den spätinterglazialen Phasen der eemzeitlichen Molluskensukzessionen aus thüringischen Travertinen nachgewiesen, so zum Beispiel von Taubach und Burgtonna (MANIA 1975, 1978). Sie sind in die Zeit kurz nach der optimalen Entwicklung der Sukzessionen, also nach der durch die Helicigona banatica-Fauna gekennzeichneten Entwicklungsphase einzuordnen. Auch diese enthalten, wie diejenigen von Schöningen, verhältnismäßig wenige Arten, vor allem einen geringen Anteil an echten Waldarten. Neben jenen der Gruppe 1, die in der Fauna von Schöningen nachgewiesen wurden, kommen noch Acicula polita, Cochlodina laminata und Helicodonta abvoluta vor. Auch das in dieser Phase zu beobachtende Auftreten der boreo-alpinen Discus ruderatus ist kennzeichnend. Sonst sind vor allem Arten der Gruppen 2 und 3 vertreten - wie in der Schöninger Fauna. Diese Assoziation wurde als "allgemeine mitteleuropäische Waldfauna" oder Cocblodina laminata - Helicodonta obvoluta Assoziation bezeichnet. Obwohl diese beiden Arten in den Proben von Schöningen fehlen, gehört ihre Fauna zur gleichen Assoziation.

\subsubsection{Pollenanalytische Gliederung der Früh-Weichsel-Abfolge}

Das Profil war nur an der Basis pollenführend und zeigt relativ gleichmäßige Kurvenverläufe, die nicht die wechselnden Humositätsgrade des Sedimentes nachzeichnen (s. Abb. 7). 
Zone SFW $1(240-130 \mathrm{~cm})$ :

Nichtbaumpollen-Pinus-Betula-Zone

In diesem basalen Abschnitt (Abb. 7) herrschen Gräser und Sauergräser und typenreiche Kräutergesellschaften vor. Neben Pollen von Kiefer und Birke kommen noch in Spuren Fichten- und geringfügig vermehrt Erlenpollen vor. Salix- und JuniperusPollen sind ebenfalls festgestellt worden. Vereinzelt wurden Larix-Pollen gefunden. Die starke Beteiligung von Potamogeton, Cyperaceae und Sphagnen zeigt die zum Zeitpunkt der Ablagerung herrschen-

Tab. 2: Molluskenfauna des eemzeitlichen Lockertravertins nach ökologischen Gruppen differenziert.

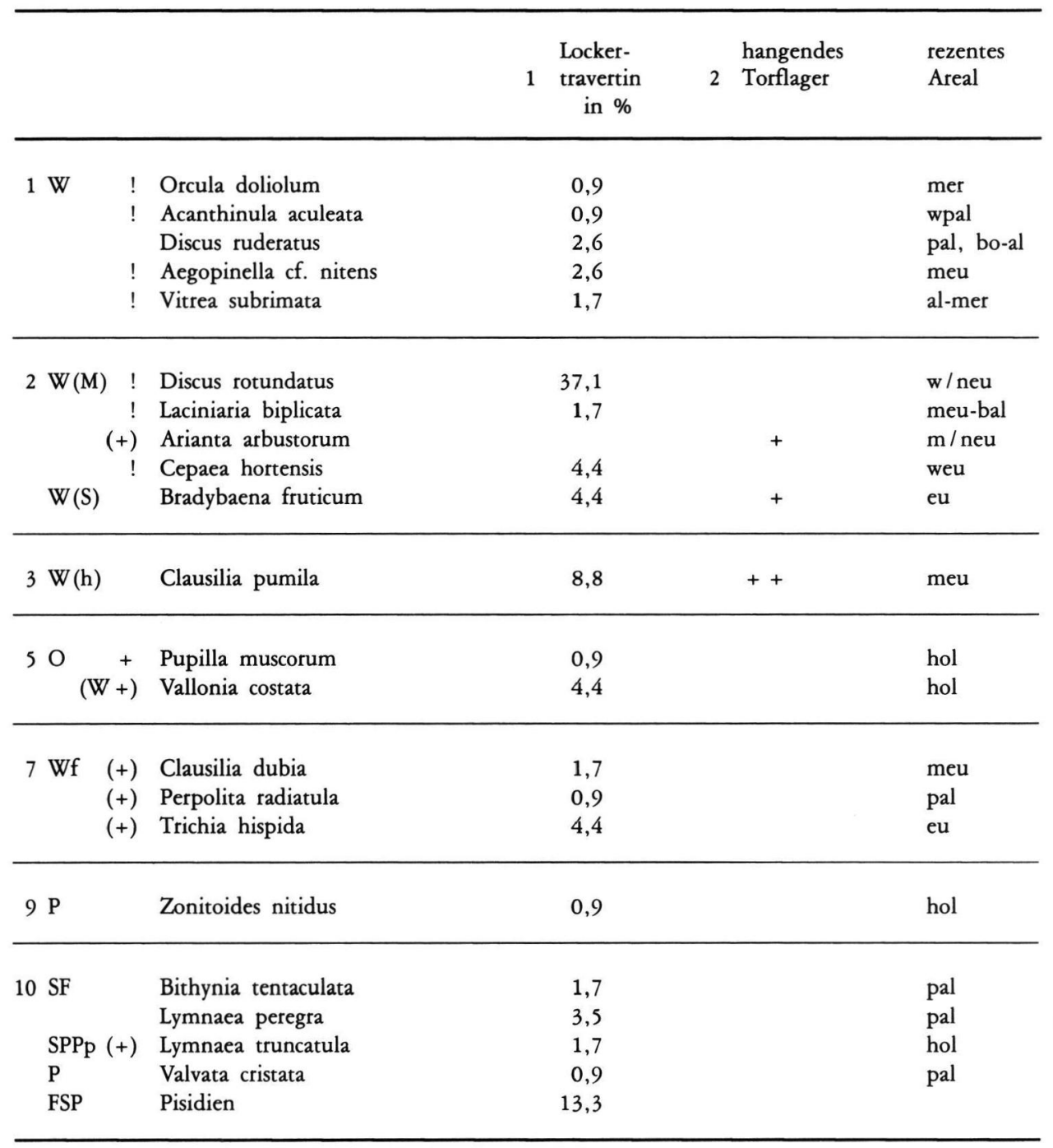

1-10 Ökologische Gruppen nach LožEK 1964: 1 ausgesprochene Waldarten, 2 vorwiegend im Wald, Gebüsch, Waldsteppe, 3 Waldarten mit hohen Feuchtigkeitsansprüchen (z. B. Auwald), 5 waldfeindliche Arten, offene Landschaft, 7 euryöke Arten an mittelfeuchten Standorten, 9 Arten mit hohen Feuchtigkeitsansprüchen, Sumpfarten, 10 Wasserarten.

Ökologische Kennzeichnung: W Wald, M mittelfeuchte Standorte, W(h) feuchte bis nasse Waldstandorte, S Steppe, trockene sonnige Standorte, O offene Landschaft, $\mathrm{f}$ feuchte Standorte, S stehendes Wasser, F fließendes Wasser, P Sumpf, Pp periodischer Sumpf,

Klimawert: ! bezeichnende Arten der Warmzeit, + auch im Löß, (+) akzessorisch im Löß. 


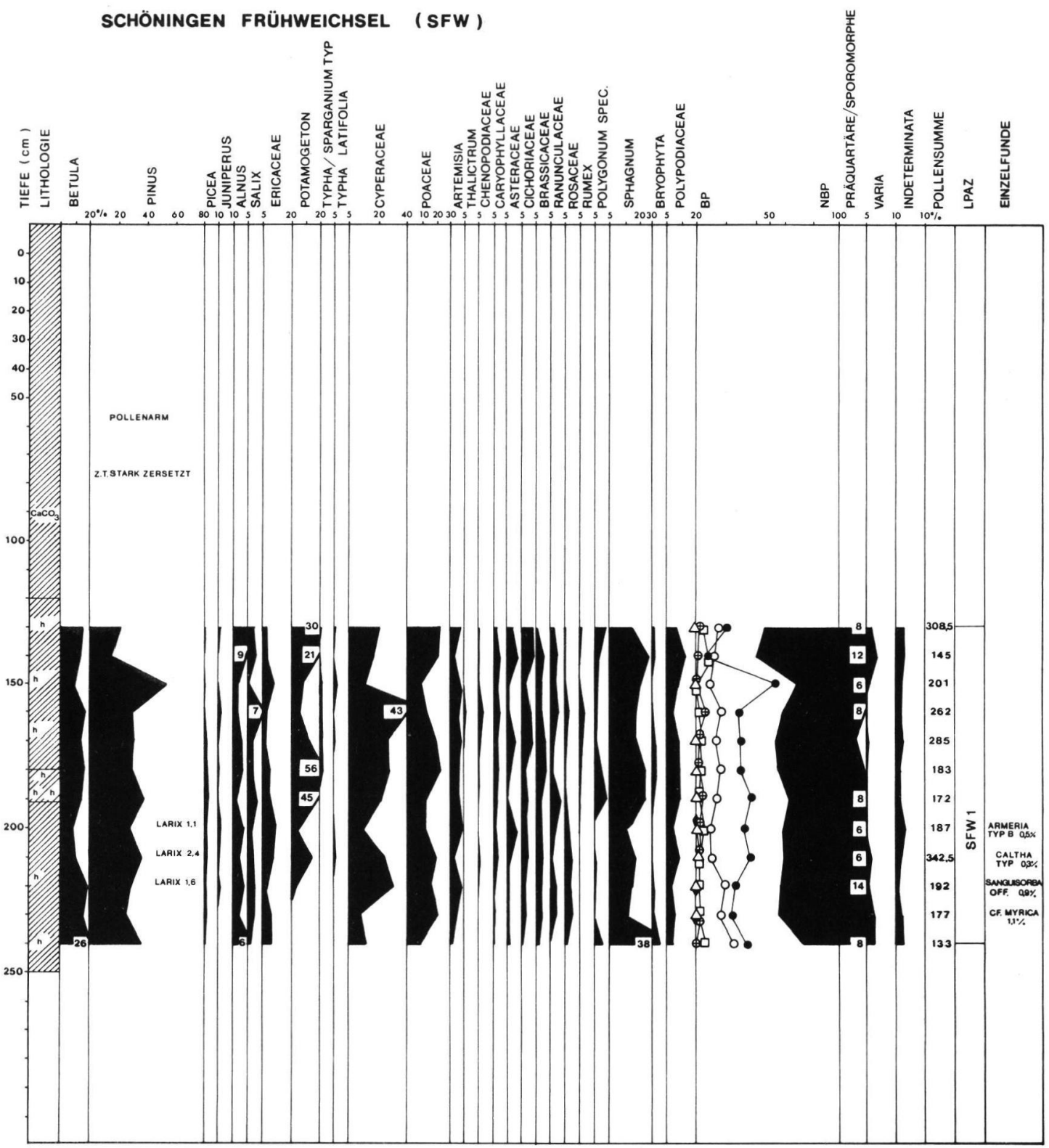

Analyse: B. Albrecht, B. Urban 1989

Abb. 7: Pollendiagramm des Früh-Weichsel.

den feuchten Bedingungen an einem Gewässerrand mit versumpften Zonen. Innerhalb der vermessenen Betula-Pollenkörner kommen zwischen $150 \mathrm{~cm}$ und $190 \mathrm{~cm}$ mit wechselnden Anteilen auch stets die kleinen Pollenformen vor, weshalb mit gewisser Vorsicht auf das Vorkommen von Betula nana auch in diesem Abschnitt geschlossen werden kann.

\subsubsection{Diskussion der Vegetations- und Klimaverhältnisse}

Von dem $165 \mathrm{~cm}$ mächtigen Lockertravertin sind nur $70 \mathrm{~cm}$ pollenführend. Der basale Teil war aufgrund zu starker Zersetzung der Sporomorphen nicht auswertbar. Daher wurde das noch verbleibende Sedi- 
mentpaket in der Regel in $1-2 \mathrm{~cm}$ Abständen auf seinen Palynomorphen-Gehalt hin analysiert. Es zeigten sich wechselnde Erhaltungszustände und unterschiedlich starke Pollenführung.

Das zeitliche Einsetzen der Quellkalkbildung mit scharfer Grenze zum Liegenden, wie oben beschrieben, läßt sich nicht exakt angeben. Die Pollenführung setzt mit auswertbaren Spektren erst bei $98 \mathrm{~cm}$ Tiefe inmitten der Hainbuchenphase des Interglazials ein. Diese Pollenzone (T 1a) ist mit der Zone IV des EemInterglazials von Bispingen/Luhe (MÜLLER 1974) korrelierbar. Sie liegt im hochwarmzeitlichen, ca. 4000 Jahre umfassenden Klimax des Eem-Interglazials. Es folgt mit der Zone T 1b eine Kiefern-Hainbuchenreiche Zeit, die der Zone Va nach MÜLLER (1974) entspricht. Die Tannen-Kiefern-Fichtenzeit erstreckt sich über die Abschnitte T $2 a$ und T 2b, entsprechend der Pollenzone Vb nach MÜLleR (1974). Die Travertinbildung endet mit dem Abschnitt T 3, der bereits von der Kiefer beherrscht wird und während dem sich Hochmoorverhältnisse einstellten, die unter anderem auch das Ende des Interglazials deutlich machen. Gestützt auf die Jahresschichtenzählungen an eemzeitlichen Kieselgurvorkommen (MỨLLER 1974) kann, vorsichtig geschätzt, das Bildungsalter des pollenanalytisch belegten Teils der Quellkalkbildung auf etwa 6000 Jahre veranschlagt werden.

Auffällig bleiben die hohen Tannenwerte in den Ablagerungen der interglazialen Spätphase. Das Untersuchungsgebiet, das heute ein kontinental getöntes Klima besitzt, nimmt eine in das mitteldeutsche Trockengebiet überleitende Position ein. Ein Vergleich mit der Vegetationsentwicklung des aus dem Tagebau Gröbern im Bitterfelder Braunkohlenrevier bekannten eemzeitlichen Elefantenschlachtplatzes (LITT \& WEBER 1988) zeigt jedoch eine wesentlich stärkere Hainbuchendominanz und Tannenanteile bis maximal $30 \%$ in den entsprechenden vegetationsgeschichtlichen Abschnitten des dortigen Gebietes. Es liegt daher nahe, die beträchtlichen Tannenanteile des Schöninger Profiles auf Vorkommen an collinen, die Helmstedter Lößmulde umgebenden Standorten zurückzuführen und lokale Einflüsse als Ursache für den Tannenreichtum anzusehen.

Auch die Molluskenfauna weist auf endinterglaziale Bedingungen zur Ablagerungszeit hin. Aus der Thanatozönose läßt sich eine mit Wald und Gebüschen durchsetzte Auenlandschaft rekonstruieren, die von einem kleineren Fließgewässer durchzogen und von Flachwasserbiotopen durchsetzt war.

Teilweise unruhige Sedimentationsbedingungen im Anschluß an eine Hochmoorphase charakterisieren das Ende des Eem-Interglazials von Schöningen, wo- rauf unter anderem auch vermehrt umgelagerte präquartäre Sporomorphe hindeuten, so zum Beispiel in der Pollenzone T4. In dieser Zone ist eine deutliche klimatische Verschlechterung zu erkennen, was durch die ersten kräftigen Artemisia-Gipfel (14\%) und eine starke Zunahme der Süß- und der Sauergräser, wie auch anderer lichtliebender Kräuter und der EricalesKurve angezeigt wird. Dieser Abschnitt markiert den Beginn der Weichsel-Kaltzeit. Die Pollenzone T5 ist eine Kiefern-Birken-reiche Zeit, mit nurmehr $10 \%$ Nichtbaumpollen. Sie umfaßt nur ca. $10 \mathrm{~cm}$ des gesamten Profils und ist mit zwei Proben erfaßt worden. Handelt es sich bei den ermittelten Spektren um Ausschnitte aus dem Brörup (ZAGWIJN 1961; MenKE \& TYNNI 1984; BEHRE \& LADE 1986), so wären sie im Profil Schöningen mit sehr geringer Sedimentmächtigkeit überliefert. Da auch charakteristische Sukzessionen und Florenelemente wie Larix weitgehend fehlen oder nicht hinreichend genug nachgewiesen werden konnten, kann der Abschnitt Ts zur Zeit nicht eindeutig mit dem Brörup korreliert werden. Abschnitt T 6 hingegen zeigt wiederum mit $50 \%$ Kräuteranteilen, im wesentlichen Poaceae, Artemisia und Caryophyllaceae, kaltzeitliche Verhältnisse auf.

Die im Hangenden des Lockertravertins ausgebildete Abfolge mehr oder weniger humoser Schluffe ist während eines frühweichselzeitlichen Stadials gebildet worden. Die Ablagerungen sind nur in der unteren Profilhälfte pollenführend und ließen keine Differenzierung zu. Da sie unmittelbar mit dem Liegenden verknüpfbar waren, läßt sich das Profil SFW konkordant an die Travertinabfolge anschließen.

\section{Ausblick}

Seit Beginn der kontinuierlichen Bearbeitung der Quartärabfolgen in den Tagebauen der BKB AG Helmstedt wurden im Tagebau Schöningen oberhalb der elsterzeitlichen Liegendserie dre i I n t e r g l a z i a le nachgewiesen. Dabei ist das S c h ö $\mathrm{n}$ i n g e n - In t e r g l a $\mathrm{z}$ i a 1 zwischen die eemzeitliche Lockertravertinabfolge und die holsteinzeitliche Serie (URBAN, THIEME \& ELSNER 1988) geschaltet. Der Torf des Schöningen-Interglazials befindet sich im Liegenden der Drenthe-zeitlichen Grundmoräne. In seinem Hangenden folgen zwei interstadiale, saalezeitliche Nachschwankungen (B ü d de nstedt-Interstadial I und II).

Die limnisch-telmatischen oder wiesenmergelartigen Ablagerungen der Interglaziale haben im Untersuchungsgebiet jeweils einen ähnlichen Sedimentationsbeginn. So wurden die bisher analysierten ehemaligen Bachrinnenausschnitte erst während der hoch- und teilweise sogar erst spätwarmzeitlichen Phasen ver- 
füllt, und Torf- oder Bruchwaldbildung setzte in der Regel während der interglazialen Klimaxstadien ein. Demgegenüber sind die spätinterglazialen Vegetationsabschnitte und die Übergänge in das jeweils nachfolgende Glazial mit Interstadialen in der Regel gut überliefert.

\section{Danksagung}

Die Untersuchungen im Tagebaugebiet der BKB AG Helmstedt werden seit 1988 zusätzlich durch die Deutsche Forschungsgemeinschaft dankenswerterweise großzügig gefördert.

Herm Dr. Hartmut Thieme und Herrn Peter Pfarr, Institut für Denkmalpflege, (Hannover), danken wir für Hilfe und Koordination bei Geländearbeiten und besonders für wertvolle Diskussionen bei der Fertigstellung des Manuskriptes sowie für Unterstützung bei der Erstellung von Graphiken.

Wir danken der BKB AG Helmstedt für die Möglichkeit, in den Tagebauen arbeiten zu können und für das zeitweilige, großzügige Bereitstellen von Hilfsgeräten (Bagger u.s.w.).

Für die Arbeitsmöglichkeit im Archäobotanischen Labor des Instituts für Urgeschichte und Jägerische Archäologie der Universität Tübingen sind wir ebenfalls sehr zu Dank verpflichtet.

Für die Mithilfe im Labor und für die sorgfältige Bearbeitung des Probenmaterials danken wir Frau CHRISTIANE HILMER, Fachhochschule Nordostniedersachsen (Suderburg).

\section{Schriftenverzeichnis}

BeHRE, K.-E. \& LADE, U. (1986): Eine Folge von Eem und 4 Weichsel-Interstadialen in Oerel/Niedersachsen und ihr Vegetationsablauf. - Eiszeitalter und Gegenwart, 36: 11-36; Stuttgart.

BEHRE, K.-E. (1989): Biostratigraphy of the last glacial period in Europe. - Quat. Science Rev., 8: 25-44; London.

Dickson, J. H. (1973): Bryophytes of the Pleistocene. The British record and its chorological and ecological implications. 255 S.; Cambridge University Press.

ElSNER, H. (1987): Das Quartär im Tagebau Schöningen der Braunschweigischen Kohlenbergwerke AG, Helmstedt. - Diplomarbeit, 126 S. - Institut für Geologie und Paläontologie der Universität Hannover. [unveröff.].

ERDTMAN, G. (1960): The acetolys method. — Svensk. bot. Tidskr., 54: 561-564; Stockholm.

Geissler, P. \& Zoluer, H. (1978): Paludella squarrosa (Hedw.) Brid. an der Südgrenze ihrer Verbreitung,
Charakterart einer neuen Assoziation des SphagnoTomenthypnion Dahl. - Candollea, 33: 299-319; Basel.

Hartmann, T. (1988): Elster- bis Saale-zeitliche Sedimente im Tagebau Schöningen der Braunschweigischen Kohlen-Bergwerke AG, Helmstedt. - Diplomarbeit; 153 S. - Institut für Geologie und Paläontologie der Universität Hannover. - [unveröff.].

HuCKRIEDE, R. (1967): Neues zur Geologie des Elms (Niedersachsen). - Geologica et Paleontologica, 1: 87-95; Marburg.

KOERT, W. \& DiEnemanN, W. (1927): Erläuterungen zur Geologischen Karte von Preußen und benachbarten deutschen Ländern 1:25000; Blatt Hötensleben. 88 S.; Berlin (Preuß. geol. Landesanstalt).

LeNHARD, R. (1989): Schichtlagerung und Zusammensetzung Elster- bis Saale-zeitlicher Sedimente im Baufeld Esbeck, Tagebau Schöningen, der Braunschweigischen Kohlen-Bergwerke AG, Helmstedt. - Diplomarbeit, 125 S. - Institut für Geologie und Paläontologie der Universität Hannover. - [unveröff.].

LITT, T. \& WeBER, T. (1988): Ein eemwarmzeitlicher Waldelefanten-Schlachtplatz von Gröbern, Gräfenhainichen. - Ausgrabungen und Funde, 33; 4: 181188; Berlin.

LOŽEK, V. (1964): Die Quartärmollusken der Tschechoslowakei. - Rozpravý Ústředního Ústavu Geologického, 31: 374 S.; Praha.

LUUTGE, H. (1984): Stratigraphie und Tektonik am SüdwestRand des Tagebaues Alversdorf. - Diplomarbeit und Kartierung: 48 S.; Technische Universität ClausthalZellerfeld. - [unveröff.].

MANIA, D. (1973): Paläoökologie, Faunenentwicklung und Stratigraphie des Eiszeitalters im mittleren Elbe-Saalegebiet auf Grund von Molluskengesellschaften. - Geologie, Beiheft 78/79: 175 S.; Berlin.

- (1975): Zur Stellung der Travertinablagerungen von Ehringsdorf im Jungpleistozän des nördlichen Mittelgebirgsraumes. - Abh. d. Zentr. Geol. Inst., 23: 571589; Berlin.

- (1978): Die Molluskenfauna aus den Travertinen von Burgtonna in Thüringen. - Quartärgeologie, 3: 6985; Berlin.

Menke, B. \& TynNI, R. (1984): Das Eeminterglazial und das Weichselfrühglazial von Rederstall/Dithmarschen und ihre Bedeutung für die mitteleuropäische Jungpleistozängliederung. - Geol. Jb., A 76: $120 \mathrm{~S}$; Hannover.

MERKT, J. (1986): Bericht über die Befahrung eines EemAufschlusses in der Westwand des Tagebaus Schöningen der Braunschweigischen Kohlenbergwerke, Helmstedt. - Archiv Nieders. L.-Amt Bodenfosch., ArchivNr.: 99004: 6 S.; Hannover. 
MƯLLER, H. (1974): Pollenanalytische Untersuchungen und Jahresschichtenzählungen an der eemzeitlichen Kieselgur von Bispingen/Luhe. - Geol. Jb., A 21: 149-169; Hannover.

RYBNIČEK, K. (1966): Glacial relicts in the Bryoflora of the Highlands Ceskomoravsk-Vrchovina: their habitat and cenotaxonomic value. - Folia Geobot. Phytotax. 1: 101-119; Praha.

Thieme, H., MaIER, R. \& Urban, B. (1987): Archäologische Schwerpunktuntersuchungen im Helmstedter Braunkohlerevier (ASHB). Zum Stand der Arbeiten 19831986. - Archäologisches Korrespondenzblatt, 17: 445-462; Mainz.
Urban, B., Thieme, H. \& Elsner, H. (1988): Biostratigraphische, quartärgeologische und urgeschichtliche Befunde aus dem Tagebau „Schöningen”, Ldkr. Helmstedt. — Z. dt. geol. Ges., 139: 123-154; Hannover.

ZAGWIJN, W. (1961): Vegetation, climate and radiocarbon datings in the Late Pleistocene of the Netherlands, Part I: Eemian and Early Weichselian. - Mededelingen Geol. Stichting, N.S. 14: 15—45; Maastricht.

Manuskript eingegangen am 11. 06. 1990 